\title{
Trust and Reciprocity: Foundational Principles for Human Subjects Imaging Research
}

Can. J. Neurol. Sci. 2007; 34: 3-4

There is no greater asset to human subjects research than human subjects themselves. While curiosity, the lure of a monetary incentive or a keepsake brainscan, or the occasional hope for a medical explanation for an undisclosed complaint may underly a subject's decision to participate in research involving magnetic resonance imaging (MRI), for example, altruism is a fundamental driving force. Given the terms of the research contract between the investigator and the participant, any benefit in the form of new knowledge obtained accrues to the investigator in the short term and, if an experiment is successful and has translational potential, possibly to society in the long term. Other than possible psychological benefits and a sense of worthiness, ${ }^{1}$ direct benefits to the participant are not expected. This is a feature of the investigator-subject relationship that must be conveyed by the investigator in verbal and written consent. Participant's altruism, coupled with professional responsibility and professional codes of ethics, therefore, make trust and reciprocity foundational principles in the scientific process. One important aspect of promulgating these principles is full disclosure of risks of the research, as discussed by Marshall et al for MRI in this volume.

Magnetic resonance imaging, with its excellent signal to noise ratio and flexible tissue contrast brought clinical diagnosis to new heights in the 1980s. The MRI also quickly transformed research imaging with the ability to tap anatomy noninvasively and repeatedly and, in 1990s, brought functional imagingmethods which measure changes in blood oxygenation in response to discrete stimuli - to the foreground. ${ }^{2}$ Research Ethics Boards (REBs) variously classify research with MRI as minimal to moderate risk, depending in part on the use of contrast agents, sedation, and the age and vulnerability of the population. There are known risks to human subjects that merit caution. For example, claustrophobia, metal implants, sensitivities to particular stimuli (spanning the range of olfactory to emotionally charged stimuli), and certain electronic devices such as stimulators and pacemakers are contraindications to participation in an MRI study. Other risks are more speculative and are "known unknowns." Some notable examples are the long-term effects of chronic exposure to magnetic fields as high at 9.4T, and the effects of MRI on Fetuses. Marshall et al bring together a discussion of many of these variables focused on structural imaging under the one roof of their paper. In the analysis of these risks, the authors raise important questions about current and future challenges of disclosure.

At the present time, there is no empirical answer to the question of whether exposure to magnetic fields of any field strength for human experimental purposes constitutes any real risk. Therefore, while this may be a possible risk, it is not a potential risk either for the healthy adult or child who gives assent. For the pregnant woman, avoiding such exposure might be viewed as one wise precautionary step among many to promote the healthy pre- and post-natal development of an offspring. Again, in the absence of data suggesting otherwise, participation in an MRI experiment cannot be portrayed as outrightly detrimental to health, as would for example, excessive alcohol or illicit drug use during pregnancy. Indeed, many advances in MRI - both structural and functional fetal MRI rely on the good will of pregnant mothers for the realization of this research and the eventual benefit they will bring to early diagnosis of disorders such as fetal growth retardation and preterm care. . $^{3,4}$

What then are the implications for disclosure? It is certainly imperative to proceed with disclosure during the process of consent that delineates well-researched, documented risks ("known risk"). It is even appropriate, as in the case of unexpected clinical findings in research for example, to cite studies of incidence rates and risks of false positives. ${ }^{5}$ Disclosure about unknowns should be approached with extra caution. The Office of Human Research Protections of the United States Dept of Health and Human Services (http://www.hhs.gov/ohrp/ humansubjects/assurance/consentckls.htm) states that subjects are entitled to information that they should reasonably want to know (e.g., risks or discomforts). Adjudicating entitlement may not be entirely straightforward, however, given that investigators must determine reasonable risk not only from a scientific point of view but from the purview of a subject or guardian with possibly limited relevant expertise. "Absolute safety" or "absolute certainty" rarely exists, and certainly not in science that involves advanced technology. Disclosure of some unknown risks may impose unnecessary burdens on participants, such as to create unwarranted fear, a reluctance to enroll, or compromise autonomy in decision-making. ${ }^{6}$ But indeed, what if a new risk is identified, was not initially mentioned in consent, and actually caused some harm? How do we manage already cumbersome and poorly understood consent forms in the face of increasing unknowns naturally associated with rapid innovation?

The solutions to these important questions are not simple, but the evidence to date suggests that MRI researchers have been conducting human imaging studies responsibly with respect to research ethics thus far. A rudimentary search of PubMed, with search terms such as "magnetic resonance imaging and MRI", yields more than 175,000 MRI studies of structure conducted around the world. Among these studies, approximately 66,310 concern the brain (8700 are functional brain MRI studies) for 
example, 8065 concern the heart, and 1384 concern the musculoskeletal system. Assuming a modest six subjects per study, we estimate roughly a million participants overall. To our knowledge there are no reports of MRI studies closed due to violations in the protection of human subjects either in consent or procedure, investigator misconduct during execution of imaging protocols, or legal action against an investigator or institution.

Increasing complexities of technique and application will inevitably accompany increasing complexities of disclosure. Ongoing, coordinated efforts among the neurological sciences, health law and biomedical ethics communities are needed to ensure that fairness both to participants and to the research enterprise is met, and that benefits of this powerful tool continue to be realized.

J. Illes and V. Chin Palo Alto, California, USA

\section{ACKNOWLEDGEMENTS}

The authors thank David Magnus and Gary Glover for helpful discussions. Supported by NIH/NINDS RO1 \#NS045831.

\section{REFERENCES}

1. Seelig BJ, Dobelle WH. Altruism and the volunteer: psychological benefits from participating as a research subject. ASAIO J. 2001;47:3-5.

2. Moseley ME, Glover GH. Functional MR imaging. capabilities and limitations. Neuroimaging Clin N Am. 1995;5:161-91.

3. Bangert BA. Magnetic resonance techniques in the evaluation of the fetal and neonatal brain. Semin Pediatr Neurol. 2001;8:74-88.

4. Glenn OA, Barkovich J. Magnetic resonance imaging of the fetal brain and spine: an increasingly important tool in prenatal diagnosis. AJNR Am J Neuroradiol. 2006;27(pt 2):1807-14.

5. Illes J, Kirschen MP, Edwards E, et al. Incidental findings in brain imaging research. Science. 2006;311:783-4.

6. Edwards A, Elwyn G, Gwyn R. General practice registrar responses to the use of different risk communication tools in simulated consultations: a focus group study. BMJ. 1999;319:749-52. 